\title{
A Method of Wide-Band Signal DOA Estimation Based on Multi-Sensor Underwater Array
}

\author{
Li Tingting \\ Science and Technology on Information Systems Engineering Laboratory \\ orangeltt@163.com
}

Keywords: DOA (direction-of-arrival) estimation; fourth-order cumulants; fractional Fourier transform

\begin{abstract}
The LFM signal is commonly used in both radar and sonar system. However, the steering vector of the LFM signal is changed with time, traditional DOA ectimation method cannot be used. A DOA estimation method of fourth-order cumulants based on fractional Fourier transform is proposed in this paper. By this method, the uses of popular DOA estimation algorithms are extended to LFM signal. Simulation results show that the method proposed can be used both in single and multisources.
\end{abstract}

\section{Introduction}

The fractional Fourier transform is a powerful tool to deal with LFM signal. Fractional Fourier transform is evolved from traditional Fourier transform, and has been proposed in 1980 by V. Namias [1]. Based on the good aggregation in fractional Fourier transform domain of LFM signal, the fractional Fourier transform has been popular used in signal filtering and interference separation [2], time-frequency analysis [3], signal and image processing [4-5] area. The steering vector of LFM signal is single in frequency and time- independent in fractional Fourier transform domain. Therefore, traditional DOA estimation method can be used as normal narrow-band signal. Frequency segmentation and focusing are avoided by the method proposed in this paper.

\section{Algorithm}

Hypothesis, $N$ independent LFM signals, spatial orientation are $\left(\theta_{1}, \theta_{2}, \cdots, \theta_{N}\right)$, obtained by a uniform linear array with $M$ sensors were considered. The space between each sensor is $d$. Then, the receiving signal of the array can be written as:

$$
s_{i}(t)=e^{j\left(\omega_{i} t+\mu_{i} t^{2} / 2\right)} \quad i=1,2, \cdots, N
$$

$\omega_{i}$ is the center angular frequency of the receiving signal, $\mu_{i}$ is the chirp rate of the signal. Take the first array as reference, the kth sensor of the array can be written as:

$$
\begin{aligned}
x_{k}(t) & =\sum_{i=1}^{N} s_{i}\left(t-\tau_{k i}\right)+n_{k}(t) \\
\tau_{k i} & =\frac{(k-1) d}{c} \cos \varphi_{i}
\end{aligned}
$$

$\tau_{k i}$ is the time delay of the kth sensor for the ith signal. $n_{k}(t)$ is the receiving noise of the kth sensor, and the noise of each sensor are independent. Therefore, Eq. 2 can be written as:

$$
x_{k}(t)=\sum_{i=1}^{N} e^{-j\left(\omega_{i}+\mu_{i} t\right) \tau_{k i}+j \mu_{i} \tau_{k i}^{2} / 2} s_{i}(t)+n_{k}(t)
$$

The steering vector of the signal is: 


$$
A\left(\theta_{i}, t\right)=\left[\begin{array}{c}
1 \\
e^{-j\left(\omega_{i}+\mu_{i} t\right) \tau_{2 i}+j \mu_{i} \tau_{2 i}^{2} / 2} \\
\vdots \\
e^{-j\left(\omega_{i}+\mu_{i} t\right) \tau_{M i}+j \mu_{i} \tau_{M i}^{2} / 2}
\end{array}\right]
$$

From Eq. 5, $A\left(\theta_{i}, t\right)$ is time-dependent, so $A$ is time-varying. The DOA of the LFM signal cannot be estimated directly by traditional algorithm.

The fractional Fourier transform of $x(t)$ is defined as follows:

$$
X_{p}(u)=F^{p}[x(t)]=\int_{-\infty}^{+\infty} K_{p}(t, u) x(t) d t
$$

Where, $p$ is the order of the fractional Fourier transform, and $p$ can be any real number. $K_{p}(t, u)$ is the converting nuclear of the fractional Fourier transform, defined as:

$$
K_{p}(t, u)=\left\{\begin{array}{cc}
\sqrt{\frac{1-j \cot \alpha}{2 \pi}} \exp \left(j \frac{t^{2}+u^{2}}{2} \cot \alpha-t u \csc \alpha\right) & \alpha \neq n \pi \\
\delta(t-u) & \alpha=2 n \pi \\
\delta(t+u) & \alpha=(2 n \pm 1) \pi
\end{array}\right.
$$

Based on the definition of the fractional Fourier transform, the fractional Fourier transform of the receiving signal $s_{i}(t)$ of the reference sensor can be written as:

$$
s_{i 1}^{p}(u)=\sqrt{\frac{1-j \cot \alpha}{2 \pi}} \exp \left(j \frac{u^{2}}{2} \cot \alpha\right) \times \int_{-T / 2}^{T / 2} \exp \left[j \frac{t^{2}}{2}\left(\cot \alpha+\mu_{i}\right)-j t\left(u \csc \alpha-\omega_{i}\right)\right] d t
$$

Where $\alpha=p \pi / 2, T$ is the observing time of the signal.

The realization of the fractional Fourier transform is dependent on Discrete Fourier Transform. For recent years, a variety definition of discrete fractional Fourier transform (DFRFT) and fast algorithm have been proposed. A fast algorithm proposed by H. M. Ozaktas has been widely used. The processing of the fast algorithm has been discussed in many books and literatures, and it will not be repeated here.

Based on the definition of the fractional Fourier Transform, LFM signal has best aggregate characteristics in the fractional Fourier domain. From the LFM signal parameters, we can obtain the optimal energy conversion order aggregation. On the contrary, the parameter of the LFM signal can be estimated in the fractional Fourier domain by searching the corresponding peak point of $s_{i 1}^{p}(u)$. The LFM signal parameters and the theoretical peak point of $s_{i 1}^{p}(u)$ have the relationship as follows:

$$
\begin{aligned}
& \hat{\mu}_{i}=-\cot \alpha_{i} \\
& \hat{\omega}_{i}=u_{1 m} \csc \alpha_{i}
\end{aligned}
$$

Therefore, when the rotation angle is $\alpha_{i}=-\operatorname{arccot} \mu_{i}$ of the fractional Fourier transform, $s_{i 1}^{p}(u)$ has the best energy concentration.

Depending on the characteristic of the fractional Fourier transform, the receiving signal of the kth sensor also has the best energy concentration under the same order of the reference sensor. So the fractional Fourier transform of the kth sensor can be expressed as:

$$
s_{i k}^{p}\left(u_{k m}\right)=\exp \left[-j \pi\left(\tau_{k i}^{2} \sin \alpha_{i} \cos \alpha_{i}+2 u_{k m} \tau_{k i} \sin \alpha_{i}\right)\right] \times s_{i 1}^{p}\left(u_{1 m}\right)
$$

The peak of the $s_{i k}^{p}\left(u_{k m}\right)$ in $u$-domain can be expressed as:

$$
u_{k m}=u_{1 m}+\tau_{k i} \cos \alpha_{i}
$$

Time delay $\tau_{k i}$ is very small, and its squared term is negligible. Therefore, the (10) can be approximated as: 


$$
s_{i k}^{p}\left(u_{k m}\right)=\exp \left(-j 2 \pi u_{k m} \tau_{k i} \sin \alpha_{i}\right) \times s_{k 1}^{p}\left(u_{1 m}\right)
$$

The steering vector under fractional Fourier domain signal is:

$$
A_{p}=\left[\begin{array}{c}
1 \\
\exp \left(-j 2 \pi u_{1 m} \tau_{2 i} \sin \alpha_{i}\right) \\
\vdots \\
\exp \left(-j 2 \pi u_{1 m} \tau_{M i} \sin \alpha_{i}\right)
\end{array}\right] .
$$

When $u_{1 m}$ and $\alpha_{i}$ is known, the steering vector is only related to time delay. Therefore, the steering vector is only concerned with the azimuth of the LFM signal. By this way, the LFM signal can be transform into a stable single-frequency signal after the fractional Fourier transform. So the DOA of the LFM can be estimated in the transform domain.

The peaks of the LFM signal in the fractional Fourier domain can be written as follows:

$$
\begin{gathered}
x^{p}(u)=A_{p} s_{i 1}^{p}\left(u_{1 m}\right)+Q \\
x^{p}(u)=\left[x_{1}^{p}\left(u_{1 m}\right), x_{2}^{p}\left(u_{1 m}\right) \cdots, x_{M}^{p}\left(u_{M m}\right)\right]^{\mathrm{T}} \\
Q=\left[Q_{1}\left(u_{1 m}\right), Q_{2}\left(u_{1 m}\right), \cdots, Q_{M}\left(u_{M m}\right)\right]^{\mathrm{T}} \\
Q_{k}\left(u_{i m}\right)=F^{p}\left[\sum_{m \neq i}^{N} s_{m}\left(t-\tau_{k m}\right)\right]+F^{p}\left[n_{k}(t)\right]
\end{gathered}
$$

The correlation matrix of $x^{p}(u)$ can be expressed as:

$$
R_{x x}^{p}=E\left[x^{p} x^{p}\right]=A_{p} s_{i 1}^{p}\left(u_{i m}\right) s_{i 1}^{p}\left(u_{i m}\right)^{H} A_{p}{ }^{H}+E\left[Q Q^{H}\right]
$$

The fourth-order cumulants beamforming method can be extended to fractional Fourier domain, and the fourth-order cumulants matrix in fractional Fourier domain is as follows:

$$
\begin{aligned}
Q^{p}[i, j, p, q]= & \operatorname{cum}\left\{x_{i}^{p}(u), x_{j}^{p^{*}}(u), x_{p}^{p^{*}}(u), x_{q}^{p}(u)\right\} \\
& =E\left[x_{i}^{p}(u) x_{j}^{p^{*}}(u) x_{p}^{p^{*}}(u) x_{q}^{p}(u)\right]-E\left[x_{i}^{p}(u) x_{j}^{p^{*}}(u)\right] E\left[x_{p}^{p^{*}}(u) x_{q}^{p}(u)\right] \\
& -E\left[x_{i}^{p}(u) x_{p}^{p^{*}}(u)\right] E\left[x_{j}^{p^{*}}(u) x_{q}^{p}(u)\right]-E\left[x_{i}^{p}(u) x_{q}^{p}(u)\right] E\left[x_{j}^{p^{*}}(u) x_{p}^{p^{*}}(u)\right]
\end{aligned}
$$

The steering vector of the fourth-order cumulants in $u$ domain is:

$$
B_{p}=A_{p} \otimes A_{p}^{*}
$$

Using the fourth-order cumulants matrix instead of the covariance matrix, the Bartlett spectrum based on fourth-order cumulants can be written as:

$$
P_{\text {Bartlett }}^{p}=B_{p}{ }^{H} Q^{p} B_{p}
$$

Similarly, the MUSIC spectrum is:

$$
P_{\text {MUSIC }}^{p}=\frac{1}{B_{p}{ }^{H} U_{N} U_{N}^{H} B_{p}}
$$

The algorithm of fourth-order cumulants based on fractional Fourier transform takes the following steps:

1. Calculate the theoretical fractional power $p$, based on the parameters of LFM signal;

2. Search for the actual peak,and find out $u_{1 m}$ corresponding to the peak point;

3. Construct the fourth-order cumulants matrix $Q^{p}$ and the corresponding steering vector in $\mathrm{u}$ domain;

4. Repeat the above procedure, if the signal sources with different frequency components.

Compared to the classical beamforming algorithm, this LFM signal beam forming method based on fractional Fourier Transform has following advantages: 1. The frequency divided and LFM signal frequency focusing transformation can be avoided, only one-dimensional transform can realize all 
kinds of beamforming algorithm; 2. this method can be used to estimate the DOA of multiple signals, as long as the sources can be divided in the transform domain; 3. The calculation of the algorithm proposed in this paper can be realized by fast FFT algorithm, so the calculation is small.

\section{Simulation Analysis}

Simulation 1: spatial resolution analysis of LFM signal in fractional Fourier domain based on Bartlett beamforming method.

Simulation conditions: Suppose a broadband LFM signal received by a uniform linear array with 18 sensors. The normalized frequency of the LFM signal is $0.03-0.06$, pulse width is $2 \mathrm{~ms}$, the azimuth of the signal is 0 degrees, the array element spacing is half the wavelength of LFM signal corresponds to the upper limit frequency. Resolutions of LFM signal based on conventional Bartlett spectrum and fourth-order cumulants Bartlett spectrum in $u$ domain are taken as contrast.

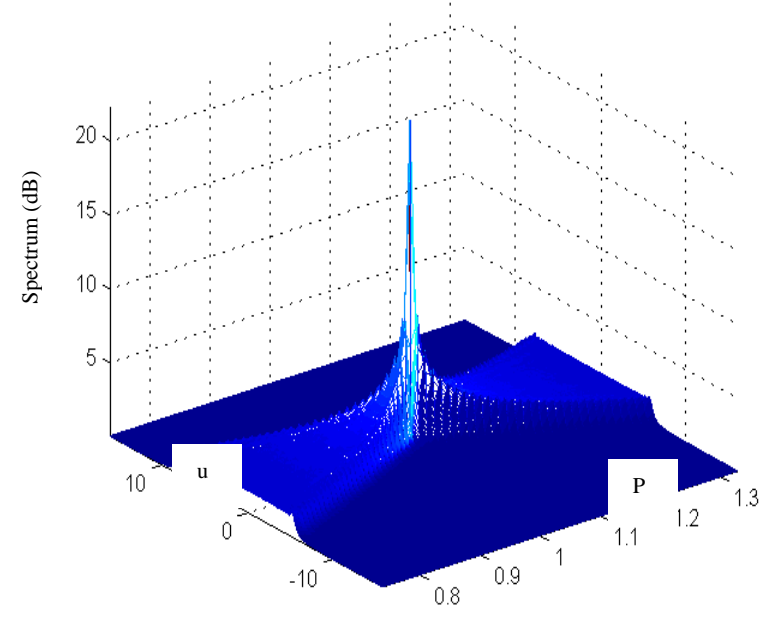

Fig. 1 Fractional Fourier spectrum

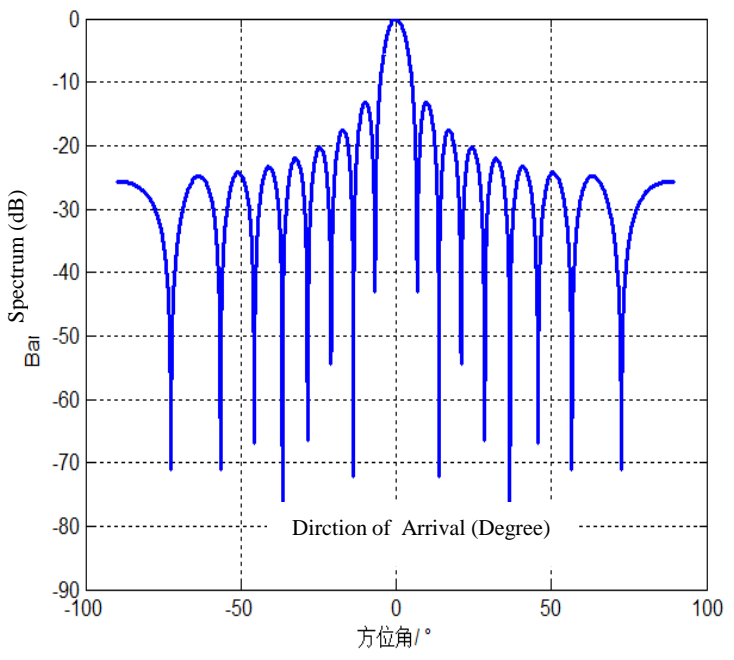

Fig. 2 Bartlett beamforming in u domain of the simulated signal

Fractional Fourier spectrum of the simulated LFM signal is shown in Figure 1. LFM signal fractional domain has a good energy concentration. Search for the peak of the fractional Fourier spectrum, and the maximum fractional Fourier power spectrum can be obtained. Bartlett spectrum based on fractional Fourier transform is shown in Figure 2. From Figure 2, the DOA of the LFM signal can be estimated by the method proposed.

Simulation 2: MUSIC spectrum estimation based on fractional Fourier transform method of two non-coherent LFM signals.

Simulation conditions: Suppose two broadband LFM signals received by a uniform linear array with 18 sensors, the signal to noise ratio of the two broadband LFM signal is 10dB. The normalized frequency of one LFM signal is 0.04-0.06, pulse width is $2 \mathrm{~ms}$, and azimuth is 5 degree. The normalized frequency of the other LFM signal is $0.02-0.06$, pulse width is $2 \mathrm{~ms}$, and azimuth is -5 degree. 


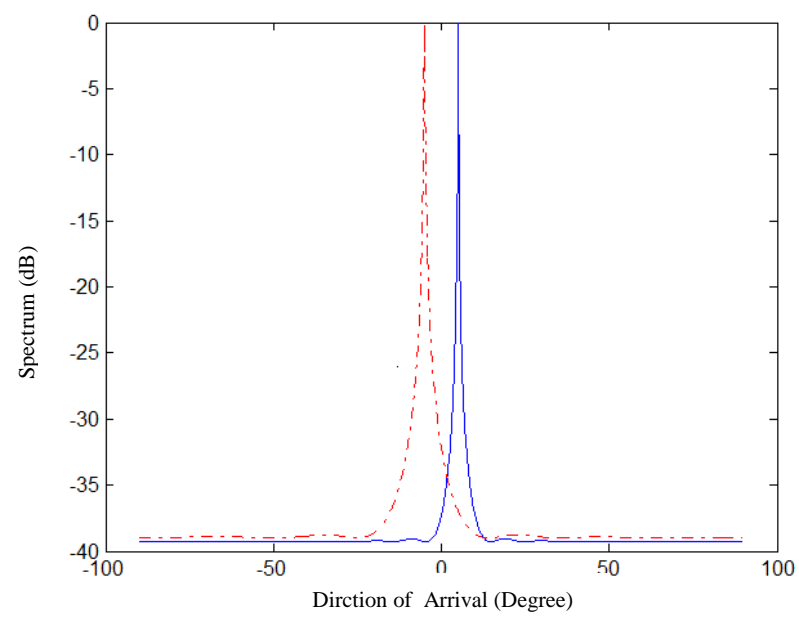

Fig. 3 MUSIC spectrum of the two LFM signals in the u domain

The MUSIC spectrums of the two LFM signals are shown in Figure 3. Although the spatial orientation of the two signals are very close, but in the fractional domain the two signals can be separated. Therefore, by the method of fractional Fourier transform, even if the two sources are very close or even coincide, the DOA of the sources can be estimated accurately as long as the signals can be separated in fractional domains.

\section{Summary}

LFM signal has the characteristic of aggregation in fractional Fourier transform domain, so the steering vector is time-independent. The fourth-order cumulants method of LFM signal DOA estimation based on fractional Fourier transform is proposed in this paper. Simulations shows that the method proposed has good characteristic in both single and multi- sources, and the uses of traditional DOA methods can be extended to LFM signal.

\section{References}

[1] Namias. The fractional order Fourier transform and its application to quantum mechanics. J. Inst. Math. APPlication, 1980(25):241-265.

[2] SHEN Li-ran, GUO Li-li, YIN Qing-bo. Fast Linear Frequency Modulation Interference Excision in DSSS System Using Fractional Fourier Transform. Science Technology and Engineering, 2014(1): 59-65.

[3] Folland G B, Nikias C L. A new positive time-frequency distribution. Proc. IEEE ICASSP, 1994:301-304.

[4] Bernardo L M, Soates O D. Fractional Fourier transforms and imaging. J.Opt.Soc.Am.A,1994,11:2622-2626.

[5] Chen Yong, Zhao Hui-Chang, Chen Si, Zhang Shu-Ning. Imaging algorithm for missile-borne SAR using the fractional Fourier transform, Acta Physica Sinica.2014(11):1-9. 